\title{
ChREBP-regulated lipogenesis is not required for the thermogenesis of brown adipose tissue
}

\author{
Chunchun Wei $\mathbb{D}^{1,5}$, Ping Wang $\mathbb{D}^{2,5}$, Qi Dong ${ }^{2}$, Xian-Hua Ma ${ }^{1}$, Ming Lu ${ }^{1}$, Shasha Qi ${ }^{1}$, Jian-Hui Shi ${ }^{1}$, Zhifang Xie ${ }^{3}$, \\ An-Jing Ren (iD ${ }^{1,4}$ and Weiping J. Zhang ${ }^{1,2}{ }^{\text {凶 }}$
}

(c) The Author(s), under exclusive licence to Springer Nature Limited 2022

\begin{abstract}
OBJECTIVES: Brown adipose tissue (BAT) plays a critical role in energy expenditure by uncoupling protein 1 (UCP1)-mediated thermogenesis and represents an important therapeutic target for metabolic diseases. Carbohydrate response element-binding protein (ChREBP) is a key transcription factor regulating de novo lipogenesis, and its activity is associated with UCP1 expression and thermogenesis in BAT. However, the exact physiological role of endogenous ChREBP in BAT thermogenesis remains unclear. METHODS: We used the Cre/LoxP system to generate ChREBP BAT-specific knockout mice, and examined their BAT thermogenesis under acute cold exposure and long-term cold acclimation. Gene expression was analyzed at the mRNA and protein levels, and lipogenesis was examined by ${ }^{3} \mathrm{H}-\mathrm{H}_{2} \mathrm{O}$ incorporation assay.

RESULTS: The mice lacking ChREBP specifically in BAT displayed a significant decrease in the expression levels of lipogenic genes and the activity of de novo lipogenesis in BAT after cold exposure, with UCP1 expression decreased under thermoneutral conditions or after acute cold exposure but not chronic cold acclimation. Unexpectedly, BAT-specific ChREBP deletion did not significantly affect body temperature as well as local temperature or morphology of BAT after acute cold exposure or chronic cold acclimation. Of note, ChREBP deletion mildly aggravated glucose intolerance induced by a high-fat diet.

CONCLUSIONS: Our work indicates that ChREBP regulates de novo lipogenesis in BAT and glucose tolerance, but is not required for non-shivering thermogenesis by BAT under acute or long-term cold exposure.
\end{abstract}

International Journal of Obesity (2022) 46:1068-1075; https://doi.org/10.1038/s41366-022-01082-7

\section{INTRODUCTION}

Global obesity rates have increased over the past $\sim 50$ years, and have reached pandemic levels [1]. Individuals with obesity often exhibit enlarged adipose tissue, increased inflammatory response, and ectopic deposition of lipids. They are also at high risk for developing complications such as type 2 diabetes, cardiovascular disease, and cancer [2-4]. Obesity is a consequence of energy imbalances and develops when energy intake exceeds energy consumption [5]. Brown adipose tissue (BAT) can burn lipids by uncoupling protein 1 (UCP1)-mediated non-shivering thermogenesis [6]. Since the discovery of BAT in human adults [7, 8], BAT activation as a potential method of treating obesity and its related diseases has become an important research topic. It has been reported that coldstimulated activation of BAT can counteract obesity and improve insulin resistance in patients with type 2 diabetes $[9,10]$.

The sympathetic system is activated under acute cold stimulation and releases catecholamine, which triggers fat mobilization and acutely activates the transcription of thermogenic genes encoding UCP1, type II iodothyronine deiodinase (DIO2), elongation of very-long-chain fatty acids protein (ELOVL) 3 , and peroxisome proliferator-activated receptor-gamma coactivator a (PGC1a). Additionally, BAT thermogenesis induced by cold exposure is associated with an increase in the expression of fuel-supplying genes involved in lipid uptake (i.e., Lipoprotein lipase, $L p l$ ), lipolysis (i.e., Hormone-sensitive lipase, $H s l$ ), and betaoxidation (i.e., Acyl-CoA Oxidase 1 and Carnitine palmitoyltransferase 1) [11-13]. As a result, increases in lipid catabolism will change the size and number of lipid droplets of brown adipocytes, which can alter BAT morphology. Free fatty acids in brown adipocytes are the fuel responsible for initiating thermogenesis, while as a ligand, fatty acids can activate UCP1 function [14].

Cold stimulation increases the expression of de novo lipogenesis (DNL) genes in BAT, including ATP Citrate Lyase (Acly), fatty Acid Synthase (Fasn), acetyl coenzyme A carboxylase 1 (Acc1), and Stearoyl-CoA desaturase $1(S c d 1)$. This is correlated with Ucp 1 expression $[15,16]$. DNL is regulated by the carbohydrate response element-binding protein (ChREBP) in BAT [17]. Cold exposure can induce a significant increase in ChREBP expression in mouse BAT [16, 18], while ChREBP expression is positively correlated with UCP1 in human BAT [16]. These reports indicate that ChREBP can promote BAT thermogenesis. However, the BATspecific overexpression of ChREBP-beta, which is a constitutively active form of ChREBP, inhibits BAT thermogenesis by downregulating UCP1 and mitochondrial function [18].

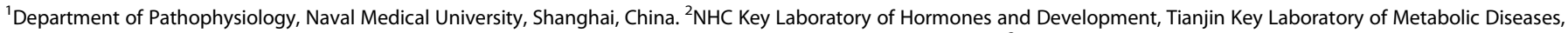

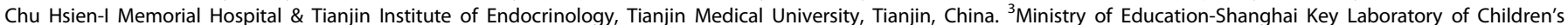

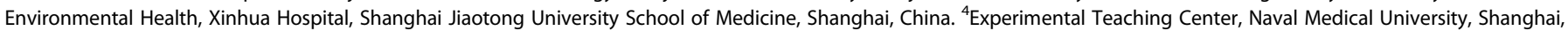
China. ${ }^{5}$ These authors contributed equally: Chunchun Wei, Ping Wang. ${ }^{凶}$ email: renanjing@smmu.edu.cn; wzhang@smmu.edu.cn 
In the present study, ChREBP deletion in BAT significantly decreases UCP1 protein levels but does not affect the rectal temperature or BAT surface temperature of mice under acute or long-term cold exposure. Interestingly, ChREBP deletion in BAT mildly aggravates the glucose intolerance induced by a high-fat diet. In summary, our work provides a comprehensive understanding of the role of BAT ChREBP in temperature maintenance and metabolic homeostasis.

\section{MATERIALS AND METHODS}

\section{Mice experiments}

All animal experiments were approved by the Animal Ethics Committee of Naval Medical University (Shanghai). Mice were housed in a pathogen-free container at a temperature between $23^{\circ} \mathrm{C}$ and $25^{\circ} \mathrm{C}$ at $12 \mathrm{~h} / 12 \mathrm{~h}$ light/dark cycle. BAT-specific ChREBP knockout mice were generated by crossing ChREBP flox/flox mice [19] with Ucp1-Cre transgenic mice [20]. All mice were freely provided with access to water and a standard chow diet or high-fat diet (HFD, 20.1\% carbohydrate, $59.9 \%$ fat, $20.0 \%$ protein in energy). Male knockout mice and littermate control were used for the experiments.

Acute cold exposure. Young mice (2-3 months) or old mice (1-year-old) were placed in a cold incubator $\left(4^{\circ} \mathrm{C}\right.$, no food) for $6 \mathrm{~h}$. Their body temperature was measured with a rectal probe, while the BAT surface temperature was measured with a thermocamera (T540, FLIR).

Long-term cold acclimation. The mice were placed in a cold incubator, and the temperature gradually decreased from $24^{\circ} \mathrm{C}$ to $4{ }^{\circ} \mathrm{C}$ over $19 \mathrm{~h}$. They were then maintained at $4{ }^{\circ} \mathrm{C}$ with free access to water and food for one month.

Glucose and insulin tolerance tests. After $6 \mathrm{~h}$ of fasting, the mice were administered gavages of glucose ( $2 \mathrm{~g} / \mathrm{kg}$ of body weight; Sigma) for the glucose tolerance test, or i.p. injection of insulin $(0.5 \mathrm{U} / \mathrm{kg}$ of body weight; Sigma) for the insulin tolerance test, as previously described [21]. Blood glucose was measured using a glucose glucometer by tail bleeding at 0,15 , 30,60 , and $120 \mathrm{~min}$ after the glucose gavage or insulin injection.

\section{In vivo lipogenesis assay}

We measured BAT DNL in vivo by using $\left[{ }^{3} \mathrm{H}\right]$-labeled water. Briefly, mice were i.p. injected with $0.5 \mathrm{ml}$ of $0.15 \mathrm{M} \mathrm{NaCl}$ containing $0.2 \mathrm{mCi}$ of $\left[{ }^{3} \mathrm{H}\right]-$ water/100 $\mathrm{g}$ body weight. One hour later, we excised BAT samples $(\sim 85 \mathrm{mg}$ per animal), heated them at $70{ }^{\circ} \mathrm{C}$ for $15 \mathrm{~min}$ in $300 \mu \mathrm{l}$ of $30 \%(\mathrm{w} / \mathrm{v}) \mathrm{KOH}$ after which $300 \mu \mathrm{l}$ ethanol was added and additional heating used for $2 \mathrm{~h}$ with occasional mixing. And then samples were acidified with $300 \mu \mathrm{l} 10 \mathrm{M}$ $\mathrm{H}_{2} \mathrm{SO}_{4}$ and extracted three times using $1 \mathrm{ml}$ petroleum ether with vortexing. The ether fractions were combined, extracted three times using $1 \mathrm{ml} \mathrm{H}_{2} \mathrm{O}$, then transferred to scintillation vials and dried for the determination of $\left[{ }^{3} \mathrm{H}\right]$-labeled fatty acids.

\section{Measurement of plasma TG/TC}

Plasma TG was measured by colorimetric assay according to Serum Triglyceride Determination Kit procedures (Sigma-Aldrich, USA). And plasma TC was measured using a Cholesterol Assay Kit (BIOSINO, China) according to procedures.

\section{mRNA expression analysis}

Total RNA was extracted from adipose tissue using the Trizol (Qiagen) method. The concentration and purity of total RNA were determined by spectrophotometry (NanoDrop; Thermo Scientific) using ratios of A260 to A280 > 1.8. Complementary DNA (CDNA) was synthesized with a First Strand cDNA Synthesis Kit ReverTra Ace-a (Code No. FSK-100, Toyobo, Osaka, Japan). Quantitative polymerase chain reaction (PCR) was performed on CDNAs by a fluorescent temperature cycler (Mastercycler Eprealplex, Eppendorf, Hamburg, Germany) with SYBR Green and specific primers for the target gene. All reactions were performed in 96-well plates with a total volume of $10 \mu \mathrm{l}$. All primers were synthesized by the Jieli Bioscientific Company (Shanghai, China). In each plate, $\beta$-actin was used as an internal control.

\section{Western blots}

Tissues were lysed in the urea lysis buffer $(25 \mathrm{mM}$ Tris-HCl, $8 \mathrm{M}$ Urea, $1 \%$ SDS, $1 \mathrm{mM}$ EDTA, $0.7 \mathrm{M} \mathrm{DTT}, \mathrm{pH}$ 7.4). After ultrasonic homogenization, centrifugation was performed at $12,000 \times g$ for $15 \mathrm{~min}$. The whole lysate was separated by $8-15 \%$ gradient SDS-PAGE gel electrophoresis and transferred onto the PVDF membrane. The membranes were incubated with the primary antibodies (Supplementary Table 1), probed with horseradish peroxidase (HRP)-conjugated anti-rabbit or anti-mouse secondary antibodies (1:5000, Vector Laboratories), developed using a Chemiluminescent Detection Kit (ECL Plus, Amersham Pharmacia Biotech), and analyzed using a Luminescent Image Analyzer (las-4000mini, Fujifilm). The target protein was then normalized to $\beta$-actin as the loading control.

\section{Histological analyses}

Samples of adipose tissue fixed in $4 \%$ paraformaldehyde were embedded in paraffin and stained with hematoxylin and eosin. For immunohistochemical analysis of UCP1, the adipose tissue sections were incubated overnight at $4{ }^{\circ} \mathrm{C}$ with rabbit polyclonal anti-UCP1 antibodies (1:1000, \#ab10983, Abcam) and then incubated with HRP-polymer conjugated antirabbit IgG $(1: 1000, \# a b 6721, A b c a m)$ at $25^{\circ} \mathrm{C}$ for $2 \mathrm{~h}$. They were then visualized with $0.05 \%$ DAB.

\section{Statistical analysis}

Sample size determination was based on previous experience with similar studies. Mice were randomly divided into groups ( $n=5-8$ per group). All tests and analyses (excluding Western blot) were performed by investigators blinded to the treatment. Only sick and/or wounded animals were excluded from the analyses. The variance was similar between compared groups. A student's $t$-test was used to compare the means between two groups of mice. Repeated-measures ANOVA was used for the acute cold exposure test. For multiple comparisons of means in different groups, a least significant difference test was used. Graphpad prism 6 and SPSS17.0 were used for statistical analysis.

\section{RESULTS}

\section{Generation of BAT-specific ChREBP knockout mice}

To evaluate the role of ChREBP in BAT, we generated ChREBP BATspecific knockout (ChBO) mice (Fig. 1A). Efficient deletion of the ChREBP gene was confirmed in the BAT from $\mathrm{ChBO}$ mice at the mRNA and protein levels by RT-PCR and western blot analysis, respectively (Fig. 1B, C). In contrast, no deletion was detected in the inguinal white adipose tissue (iWAT), epididymal WAT (eWAT), or liver from $\mathrm{ChBO}$ mice maintained at room temperature. As a result, the mRNA and protein levels of the ChREBP target genes involved in lipogenesis, such as Scd1 and Fasn, were significantly decreased in the BAT of ChBO mice compared to that of the control mice (Fig. 1D, E). This indicates that we successfully generated a mouse model with a tissue-specific knockout of ChREBP in BAT.

ChREBP deletion aggravates diet-induced glucose intolerance To examine the role of BAT ChREBP in glucose and lipid metabolism, we first phenotypically analyzed the ChBO mice. When housed at room temperature, ChBO mice that were fed a chow diet exhibited no significant differences in body weight, plasma total cholesterol, plasma triglyceride, or glucose tolerance compared with the control (Supplementary Fig. 1A-D). Furthermore, glucose tolerance was similar between $\mathrm{ChBO}$ and control mice after 1 month of cold acclimation at $4{ }^{\circ} \mathrm{C}$ (Supplementary Fig. 1E). After 12 weeks of HFD feeding, $\mathrm{ChBO}$ and control mice did not show any significant differences in their body weight, plasma total cholesterol, plasma triglyceride, or tissue mass of BAT, iWAT, eWAT, or liver (Supplementary Fig. 2A-D). However, HFD-fed ChBO mice showed an aggravation in glucose intolerance compared to control mice (Fig. 1F). While there was no significant difference in the insulin tolerance test between the two genotypes based on blood glucose levels, ChBO mice exhibited a mild decline in insulin sensitivity when the glucose levels were normalized to their initial values (Supplementary Fig. 2E). This indicates that blocking DNL in BAT could impair metabolic homeostasis. 


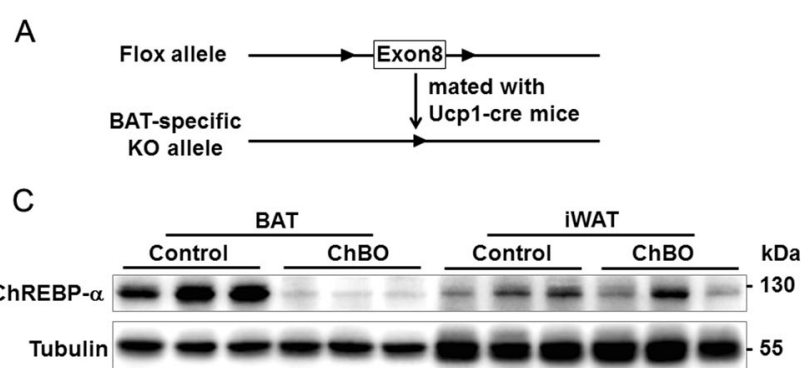

E

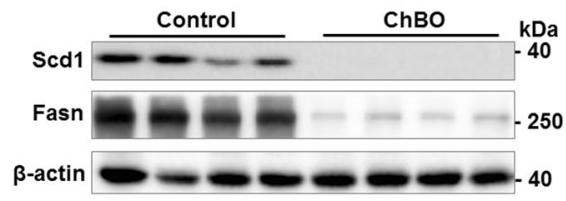

B
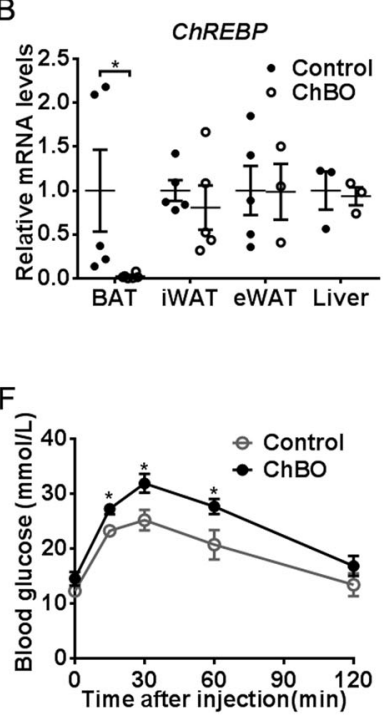
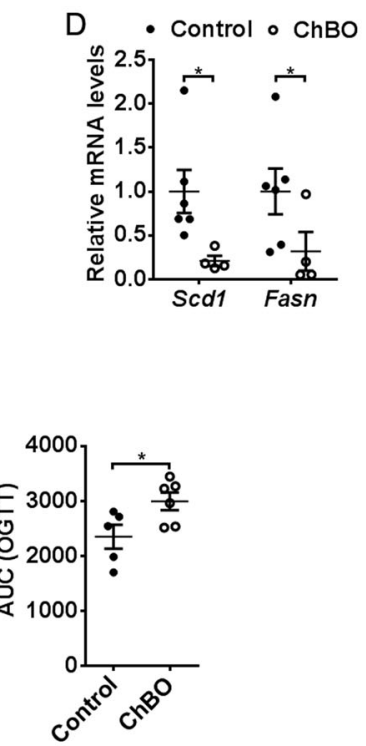

Fig. 1 Generation of BAT-specific ChREBP knockout mice. A Schematic demonstration of the generation of brown adipose tissue (BAT) specific ChREBP knockout mice. Chrebp BAT-specific knockout (ChBO) mice were generated by crossing Chrebp ${ }^{\text {flox }}$ mice with Ucp1-Cre transgenic mice. B The specific knockout of ChREBP mRNA in BAT from ChBO mice at room temperature $(n=3-5$ per group). Data are shown after normalization with $\beta$-actin. iWAT, inguinal WAT; eWAT, epididymal WAT. C Protein expression of ChREBP- $\alpha$ in BAT or iWAT from ChBO and control mice housed at room temperature ( $n=3$ per group). Tubulin was used as a loading control. D Downregulation of mRNA levels of the lipogenic target genes in BAT from $\mathrm{ChBO}$ mice housed at room temperature ( $n=4-6$ per group). E Protein expression of Fasn and Scd 1 in BAT from ChBO and control mice housed at room temperature ( $n=4$ per group). $\beta$-actin was used as a loading control. $\mathbf{F}$ Glucose tolerance test with $2 \mathrm{~g} / \mathrm{kg}$ glucose and area under the curve of HFD-fed ChBO and control mice housed at room temperature. Data are presented as mean \pm SEM. ${ }^{*} p<0.05 ;{ }^{* *} p<0.01 ;{ }^{* *} p<0.001$.

\section{ChREBP deletion in BAT does not impair cold tolerance}

To evaluate the role of BAT ChREBP in cold tolerance, we analyzed $\mathrm{ChBO}$ and control mice maintained at room temperature. The two genotypes showed similar rectal temperatures (Supplementary Fig. 3A, B) and adipose tissue masses (Fig. 2A, B, top), regardless of whether they were young or old. When exposed to acute cold conditions $\left(4^{\circ} \mathrm{C}\right)$ for up to $6 \mathrm{~h}$ with no access to food, the young mice could comparably maintained their body temperature (Fig. $2 \mathrm{~A}$, bottom), while the 1-year-old mice showed a similar defect to defend their body temperature (Fig. 2B, bottom).

For the thermogenic activity of BAT is decreased in thermoneutral environment, we tested the cold tolerance of young mice maintained at $30^{\circ} \mathrm{C}$. After being housed at $30^{\circ} \mathrm{C}$ for a month, the mice showed a dramatic decrease in their acute cold tolerance, though no difference was found in the cold tolerance and adipose tissue mass between $\mathrm{ChBO}$ and the control mice (Fig. 2C). To verify the effect of diet on the thermogenic phenotype of BAT, 10-week old mice housed at thermoneutral temperature were divided into two groups, which were then exposed to acute cold under feeding or fasting conditions. Fasting reduced the cold tolerance of mice, but both genotypes showed similar cold tolerance under both conditions (Supplementary Fig. 3C). This indicates that ChBO mice can maintain normal body temperatures as control mice when adapted to room temperature or $4{ }^{\circ} \mathrm{C}$, and showed cold tolerance similar to the control mice during acute cold exposure with or without food access.

To further clarify the effect of ChREBP on the thermogenic activity of BAT, we used a thermal camera to directly monitor the change in BAT surface temperature during acute cold exposure $\left(4{ }^{\circ} \mathrm{C}\right)$. ChBO and control mice housed at $30^{\circ} \mathrm{C}$ showed no significant differences in BAT thermal imaging and temperature after $1-2.5 \mathrm{~h}$ of acute cold exposure (Fig. 2D). This suggests that ChREBP deficiency does not affect BAT thermogenesis induced by acute cold exposure.

\section{ChREBP deletion in BAT does not impair the energy homeostasis of cold-acclimated mice}

The mechanism behind body temperature maintenance in mice could differ between long-term cold acclimation and acute cold exposure. Therefore, we also adapted mice to severe cold conditions $\left(4^{\circ} \mathrm{C}\right)$ for a month. Chronic cold-acclimated mice can survive in a cold environment $\left(4^{\circ} \mathrm{C}\right)$. After long-term cold acclimation, we detected no differences in the body weight or mass of adipose tissue between $\mathrm{ChBO}$ and control mice (Fig. 3A, $B)$. When separately housed without food access, the coldacclimated mice successfully maintained their body temperature in both groups (Fig. 3C). We also recorded the local temperature of brown fat in mice housed at $4{ }^{\circ} \mathrm{C}$ for 1 month and found no significant differences in the BAT surface thermal imaging and temperature between the $\mathrm{ChBO}$ and control mice (Fig. 3D, E).

\section{ChREBP deletion has no impact on BAT morphology}

The activation of BAT thermogenesis is accompanied by changes in the size and number of lipid droplets, manifested as changes in cell morphology. To further confirm our results, we histologically characterized the adipose tissue. Compared to BAT from $30^{\circ} \mathrm{C}$ housed mice, H\&E staining found remarkable morphological changes in BAT after $6 \mathrm{~h}$ of acute cold exposure or one month of cold acclimation at $4{ }^{\circ} \mathrm{C}$ (Fig. 4A). However, the BAT morphology did not differ between $\mathrm{ChBO}$ and control mice. This indicates that ChREBP is not required for BAT thermogenesis under acute cold exposure or long-term cold acclimation.

Downregulated expression of UCP1 in BAT from ChBO mice When the mice were housed at $30^{\circ} \mathrm{C}, \mathrm{UCP} 1$ protein levels decreased by $30-40 \%$ in the BAT from ChBO mice compared with the control mice, but there was no difference in Ucp1 mRNA levels (Fig. 5A, B). When the mice housed at $30^{\circ} \mathrm{C}$ were subjected to $6 \mathrm{~h}$ of acute cold 

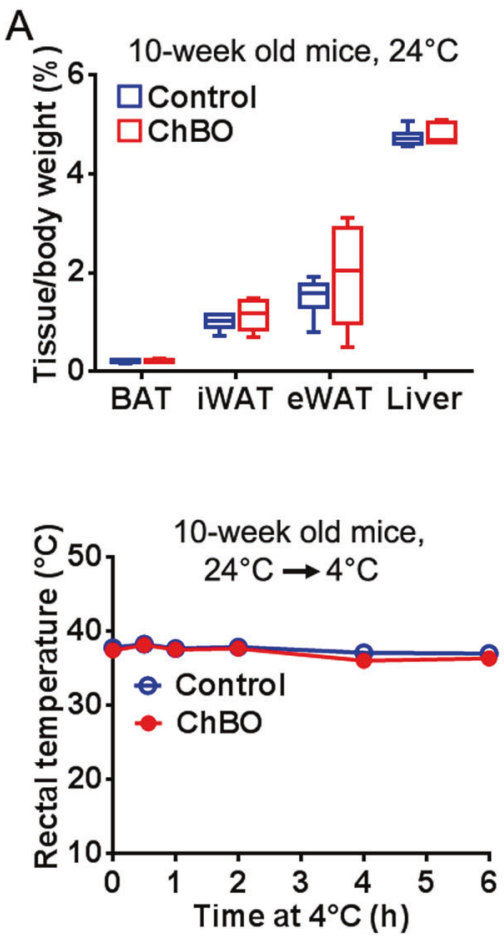
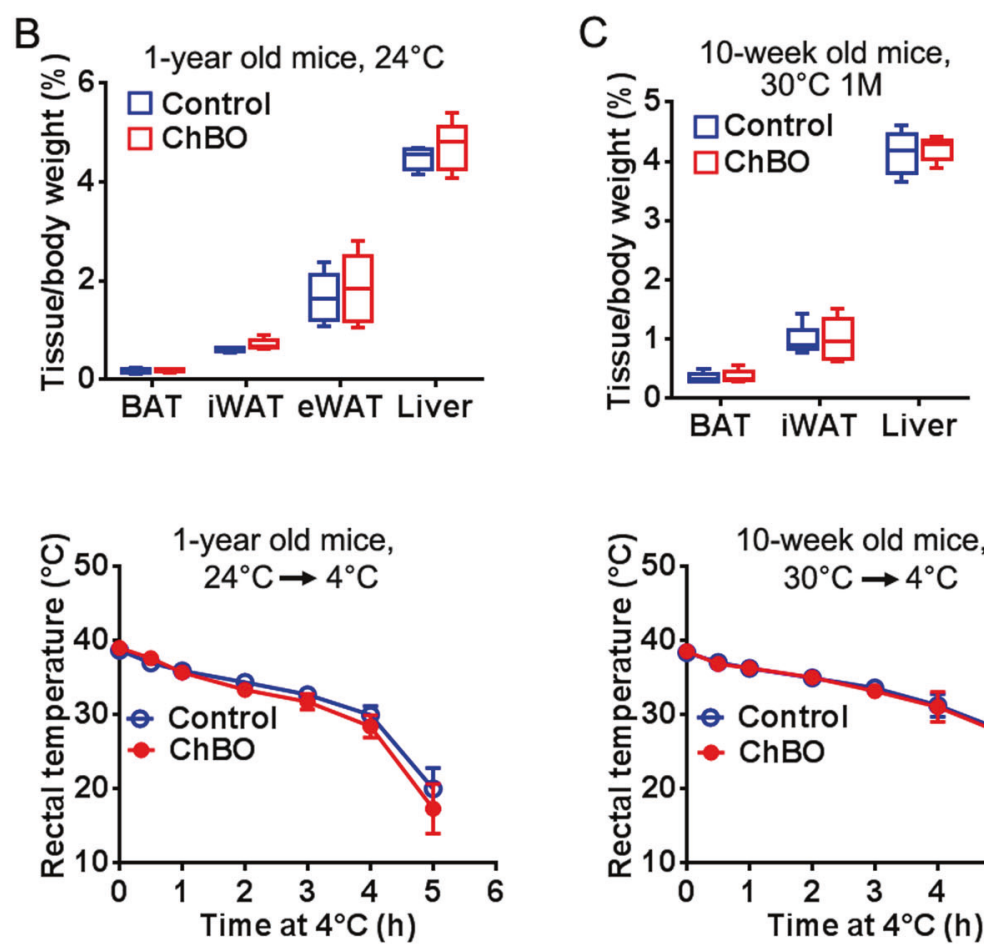

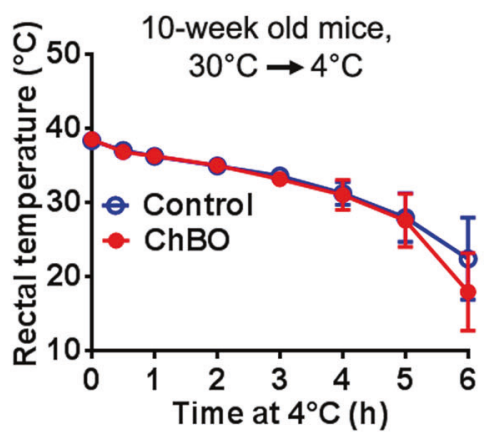

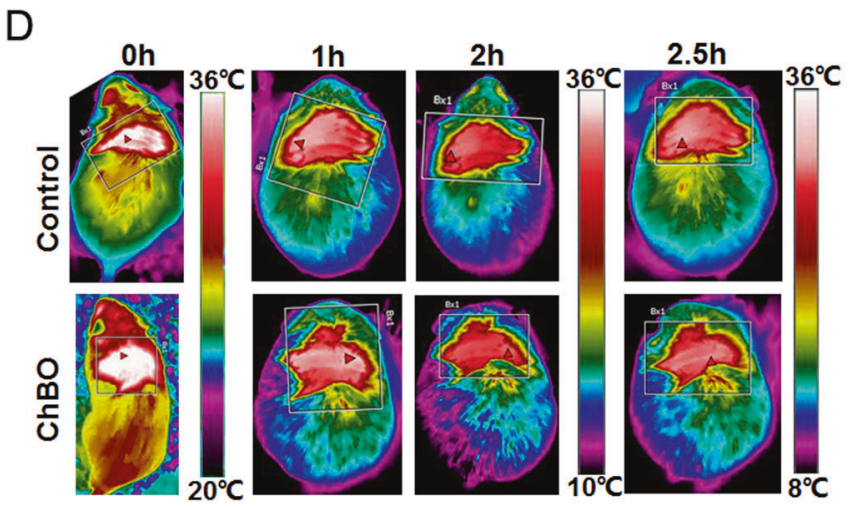

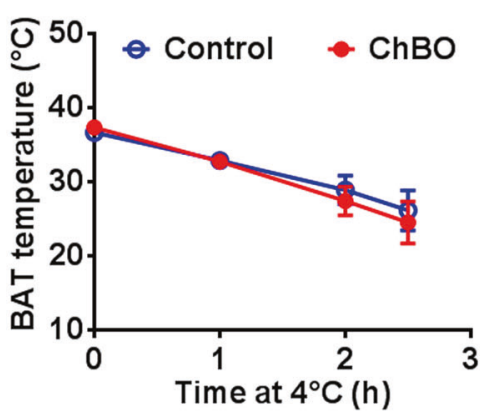

Fig. 2 ChREBP deletion in BAT does not impair cold tolerance. Chow-fed ChBO and control male mice at different ages were housed at $24^{\circ} \mathrm{C}$, and $30^{\circ} \mathrm{C}$, then subjected to cold exposure. A-C, top Tissue to bodyweight ratios of 10-week old mice housed at $24{ }^{\circ} \mathrm{C}(\mathbf{A}), 1$-year old mice housed at $24^{\circ} \mathrm{C}(\mathbf{B})$, and mice housed at $30^{\circ} \mathrm{C}(\mathbf{C})(n=5-7$ per group). A, B, bottom 10-week old mice (A) or 1-year old mice (B) housed at $24^{\circ} \mathrm{C}$ were subjected to acute cold exposure at $4{ }^{\circ} \mathrm{C}$ without food access for $6 \mathrm{~h}$ ( $n=5-7$ per group). C, bottom mice housed at $30^{\circ} \mathrm{C}$ were subjected to acute cold exposure at $4{ }^{\circ} \mathrm{C}$ without food access for $6 \mathrm{~h}(n=5-6$ per group). D Thermography and surface temperature of BAT from $\mathrm{ChBO}$ and control mice housed at $30^{\circ} \mathrm{C}$ subjected to acute cold exposure at $4{ }^{\circ} \mathrm{C}$. Images are representative of five independent experiments. Data are presented as mean \pm SEM. ${ }^{*} p<0.05 ;{ }^{* *} p<0.01$.

exposure, the mRNA and protein levels of Ucp1 both decreased in the BAT of ChBO mice compared to the control mice. When the mice were subjected to one month of chronic cold acclimation at $4{ }^{\circ} \mathrm{C}$, Ucp 1 mRNA levels decreased by $35 \%$ in the BAT of $\mathrm{ChBO}$ mice compared with control mice, but there was no difference in UCP1 protein levels. Dio2 is an important thermogenic gene that encodes deiodinase capable of catalyzing the conversion of thyroxine (T4) to the active thyroid hormone triiodothyronine (T3). Dio2 was downregulated by $50 \%$ at the mRNA level in BAT from $\mathrm{ChBO}$ mice subjected to one month of chronic cold acclimation at $4{ }^{\circ} \mathrm{C}$, though no difference was found in Dio2 protein levels. (Fig. 5A, B). The expressions of Pgcla and Elovl3 also increased following cold exposure, though there was no significant difference between the control and the $\mathrm{ChBO}$ mice (Fig. 5A, B). The protein levels of other thermogenic genes, such as PR domain containing 16 (Prdm16), were not affected by BAT ChREBP loss (Fig. 5B).

Mitochondrial respiration depends on the activity of the electron transport chain (ETC), which consists of five protein complexes (C-I to $\mathrm{C}-\mathrm{V}$ ). A proton gradient across the mitochondrial membrane created by $\mathrm{C}-\mathrm{I}$ to $\mathrm{C}-\mathrm{IV}$ drives heat production through UCP1-mediated uncoupling in brown adipocytes rather than generating ATP through C-V (ATP synthase). We investigated whether ETC composition was affected by ChREBP deletion. The subunits of respiratory chain components increased after acute and chronic cold exposure compared to the control but were not significantly changed by ChREBP loss (Supplementary Fig. 4A, B). These results indicate that ChREBP knockout does not affect mitochondrial respiratory enzymes upon cold exposure. 
A

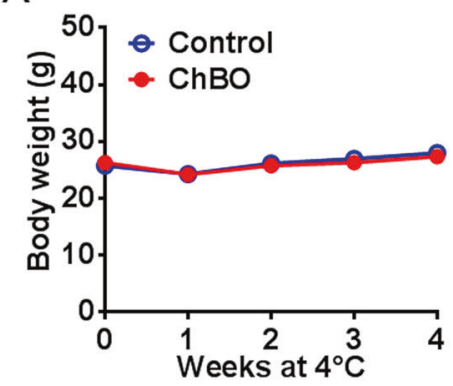

C

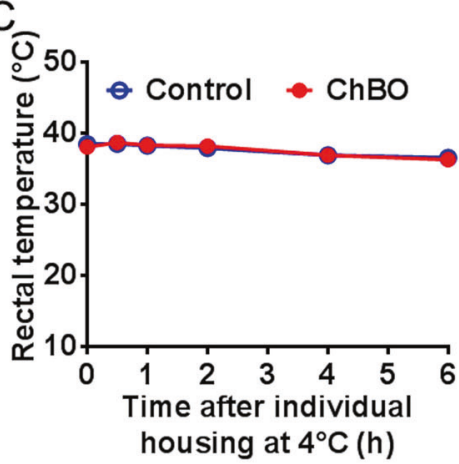

B

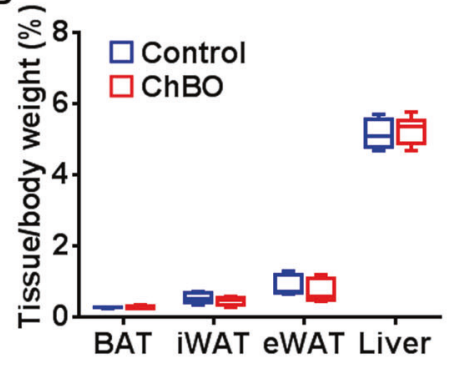

D
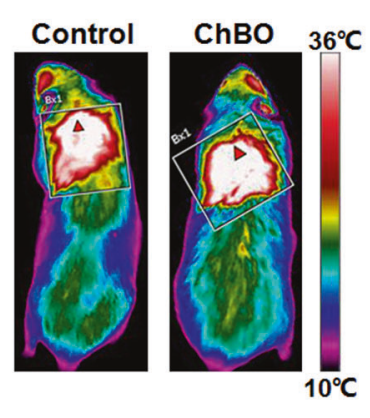

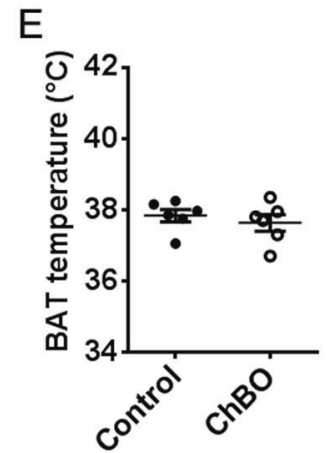

Fig. 3 ChREBP deletion in BAT does not impair the energy homeostasis of cold-acclimated mice. Chow-fed ChBO and control male mice were subjected to 1 month of chronic cold acclimation at $4{ }^{\circ} \mathrm{C}$. A Growth curve of $\mathrm{ChBO}$ and control mice. B Tissue to bodyweight ratios. $\mathbf{C}$ mice housed at $4{ }^{\circ} \mathrm{C}$ were housed in single cages without food access for $6 \mathrm{~h}$. D Thermography of BAT from ChBO and control mice. E BAT surface temperature of $\mathrm{ChBO}$ and control mice. Data are presented as mean \pm SEM ( $n=6$ per group). ${ }^{*} p<0.05 ;{ }^{* *} p<0.01$.

A

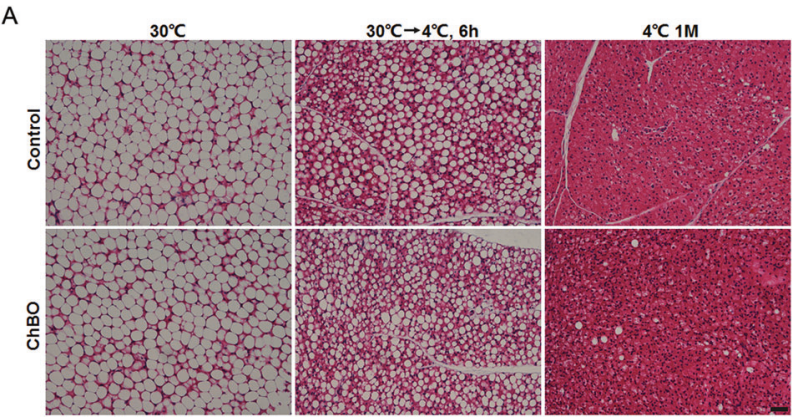

Fig. 4 ChREBP deletion has no impact on BAT morphology. A H\&E of BAT from $\mathrm{ChBO}$ and control male mice at $30^{\circ} \mathrm{C}$ and subjected to $6 \mathrm{~h}$ of acute cold exposure or 1 month of chronic cold acclimation at $4^{\circ} \mathrm{C}$. Scale bars, $50 \mu \mathrm{m}$. Images are representative of three independent experiments.

Our data indicates that while no differences were found in cold tolerance and BAT thermogenesis between $\mathrm{ChBO}$ and control mice, ChREBP deficiency significantly down-regulated UCP1 protein levels.

\section{BAT ChREBP deficiency has a significant impact on cold- induced lipid metabolism remodeling}

To determine whether the loss of ChREBP influences the fuel supply for BAT thermogenesis, we examined the expression of genes involved in lipid metabolism. Long-term cold exposure significantly induced ChREBP expression at the mRNA and protein levels, though $6 \mathrm{~h}$ of acute cold exposure did not affect ChREBP expression (Fig. 6A, B). The expression of BAT lipogenic genes was also significantly induced in both genotypes under long-term cold exposure, but the deletion of ChREBP significantly hindered the induction in BAT of ChBO mice (Fig. 6A, B, and Supplementary Fig. $5 A)$. Furthermore, we measured DNL in the BAT by injection of
${ }^{3} \mathrm{H}$-water as a tracer. After 1 week of cold-acclimation, ChBO BAT displayed a $42 \%$ reduction in the synthesis of fatty acids relative to control (Fig. 6C).

Compared to control mice housed at $30^{\circ} \mathrm{C}$, the mRNA levels of lipase (i.e., ATGL, HSL, and MGL) showed a slight but significant decrease in the control mice subjected to $6 \mathrm{~h}$ of cold exposure, while long-term cold exposure significantly and strongly induced the mRNA expression of lipase (Supplementary Fig. 6A, B). Compared to the control mice, the $\mathrm{ChBO}$ mice showed no difference in their mRNA levels or protein levels of lipase (Supplementary Fig. 6A, B). Lipid uptake-related proteins (i.e., CD36, VLDLR, and LPL) were also significantly induced in mRNA and protein levels after acute cold exposure or long-term cold acclimation, though no differences were found between the two groups (Supplementary Fig. 7A, B). Interestingly, the mRNA and protein levels of fatty acid $\beta$-oxidation genes in BAT were slightly upregulated by ChREBP deletion under acute or long-term cold exposure (Supplementary Fig. 8A, B).

In conclusion, long-term cold acclimation significantly induced de novo lipogenesis and beta-oxidation, while ChREBP deletion hindered the induction of de novo lipogenesis but mildly enhanced the expression of genes involved in beta-oxidation.

\section{ChREBP deficiency does not impair WAT browning}

Considering that chronic cold exposure induces the browning of WAT in association with UCP1 activation, which contributes to adaptive thermogenesis, we next examined the potential effect of UCP1 activation-driven ChREBP deletion on WAT browning after one month of cold exposure. ChREBP expression was decreased by $60 \%$ at the mRNA level in iWAT from ChBO mice compared to those in the control mice (Supplementary Fig. 9A). Additionally, Ucp1 expression was significantly upregulated at the mRNA and protein levels in iWAT in the control mice after 1-month cold exposure, but the upregulation was significantly inhibited in $\mathrm{ChBO}$ mice (Supplementary Fig. 9A-C, G). The expression of Dio2 and Pgc1a were also 
A

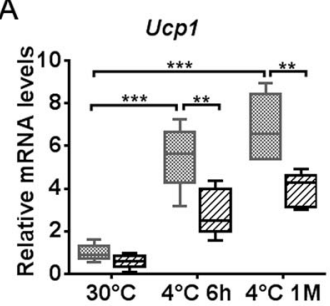

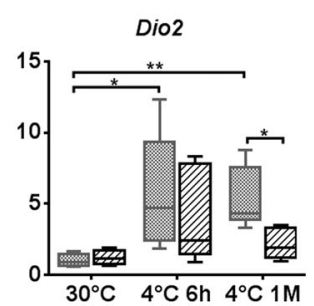
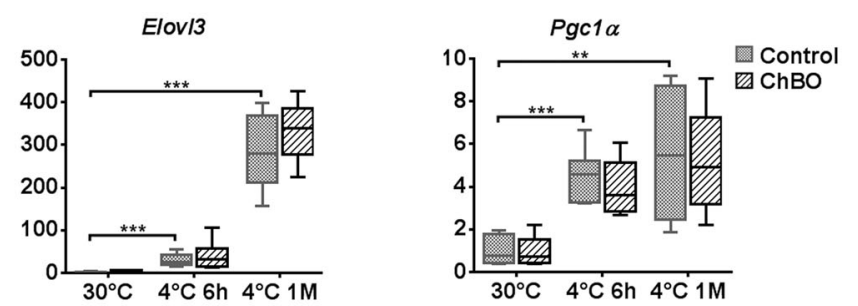

B

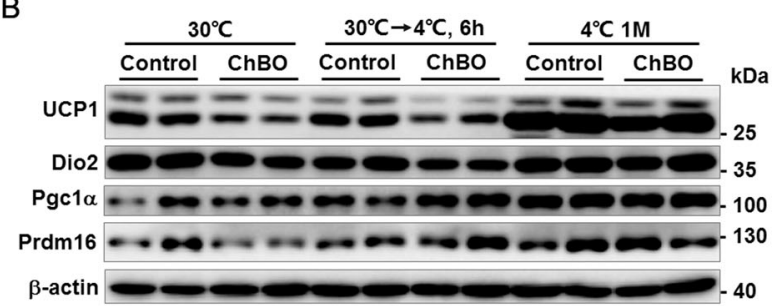

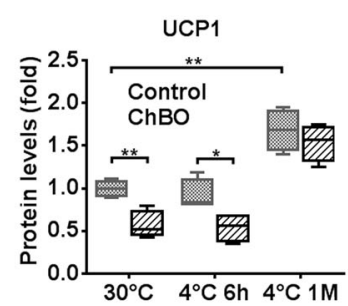

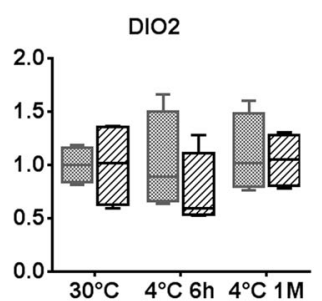

Fig. 5 Downregulated expression of Ucp1 in BAT from ChBO mice. Chow-fed $\mathrm{ChBO}$ and control male mice housed at $30^{\circ} \mathrm{C}$ were subjected to $6 \mathrm{~h}$ of acute cold exposure or 1 month of chronic cold acclimation at $4{ }^{\circ} \mathrm{C}$. A mRNA levels of Ucp 1, Dio2, Pgc1a, and Elovl3 genes in BAT ( $n=$ 6-8 per group). B Protein levels of thermogenesis-relates genes in BAT from ChBO and control mice $(n=4$ per group). Data are presented as mean \pm SEM. ${ }^{*} p<0.05 ;{ }^{* *} p<0.01 ;{ }^{* * *} p<0.001$.

induced by long-term cold acclimation at the mRNA and protein levels, though no difference was found between these two groups (Supplementary Fig. 9A-C). Furthermore, western blot analysis demonstrated that both groups expressed similar levels of proteins involved in lipid uptake (LPL) and lipolysis (ATGL, HSL) in iWAT (Supplementary Fig. 9D, E). H\&E staining analysis revealed similar iWAT morphologies in ChBO and control mice (Supplementary Fig. 9F). These results indicate that ChREBP deletion in UCP1-positive cells does not appear to affect WAT browning in response to chronic cold exposure, and proves that ChREBP loss did not impact the browning of white adipose tissue.

\section{DISCUSSION}

ChREBP- $\beta$ mRNA levels are increased by $1.5-2$-fold under cold exposure $[16,18]$, while our previous study demonstrates that ChREBP- $\beta$ overexpression sharply inhibits BAT thermogenesis [18]. These indicate a potential role of ChREBP in negatively regulating BAT thermogenesis. To explore the physiological function of ChREBP in BAT thermogenesis, we took advantage of BAT-specific knockout mice of ChREBP. Compared with control mice, ChBO mice displayed normal body weight, adipose tissue mass, body temperature, cold tolerance, as well as morphology and surface temperature of BAT under cold stress. Moreover, ChREBP deletion led to a significant downregulation of UCP1 protein levels in BAT when the mice were maintained at $30^{\circ} \mathrm{C}$ or subjected to $6 \mathrm{~h}$ of acute cold exposure. Under chronic cold acclimation conditions, despite normal induction of UCP1, ChREBP deletion hindered the induction of de novo lipogenesis and mildly enhanced the expression of genes involved in beta-oxidation.

ChBO mice displayed normal cold tolerance and BAT surface temperatures under cold stress; this phenotype was not altered by age and ambient temperature. Furthermore, there was no difference in UCP1 protein levels in BAT between the $\mathrm{ChBO}$ and control mice maintained at $4^{\circ} \mathrm{C}$ for 1 month, which suggests that ChREBP is not necessary for BAT thermogenesis. UCP1-null mice are cold intolerant due to impaired BAT thermogenesis, while heterozygous mice can retain normal body temperatures under acute cold stress [22]. In conclusion, although ChREBP deletion decreases UCP1 protein levels during acute cold stimulation, it did not affect the adaptive nonshivering thermogenesis of BAT under cold conditions.

Our finding that ChREBP deletion does not impact BAT thermogenesis differed from other reports. Joan SanchezGurmaches et al. reported that the expression of DNL genes in BAT significantly increased under mild cold stimulation and that ChREBP-mediated DNL can optimize fuel storage and thermogenesis [16]. They also reported that ChREBP- $\beta$ and Fasn expression were correlated with high UCP1 in human BAT [16]. Katz et al. pointed out that T3 and high glucose upregulated UCP1 through a ChREBP pathway [23]. In this study, DNL proteins (i.e., ACLY, FASN, SCD1, and ACC1) significantly decreased in ChBO mice but did not impair cold tolerance and BAT thermogenesis. This indicates that endogenous ChREBP-regulated de novo lipogenesis is not required for non-shivering thermogenesis. Our previous study demonstrated that ChREBP- $\beta$ overexpression in brown adipocytes impaired BAT thermogenesis and made the mice more susceptible to body temperature loss under acute cold conditions [18]. In contrast to the ChREBP- $\beta$ protein, which can be detected by constitutive overexpression, cold-induced ChREBP- $\beta$ protein expression is difficult to identify with western blot analysis using currently available antibodies under these experimental conditions [18]. Therefore, although constitutively overexpressed ChREBP- $\beta$ inhibits BAT thermogenesis, endogenous ChREBP has little impact on BAT thermogenesis. This is likely due to its relatively low activation under cold exposure conditions.

Our results also demonstrate the role of BAT ChREBP in glucose homeostasis. ChREBP was originally identified as a transcription factor regulating DNL [24], and its expression levels in WAT are associated with metabolic health $[25,26]$. Both gain-of-function and loss-of-function studies demonstrate that ChREBP-regulated DNL in adipocytes can positively affect metabolic homeostasis under the settings of overnutrition [27-29]. Our previous study demonstrated that ChREBP- $\beta$ overexpression in brown adipocytes increased DNL gene expression and improved metabolic performance under a high-fat diet [18]. In this study, ChBO showed an aggravation in glucose intolerance after 12-weeks of HFD. Our results further demonstrate that BAT ChREBP is beneficial to glucose homeostasis, which is independent of its thermogenic activity. 
A

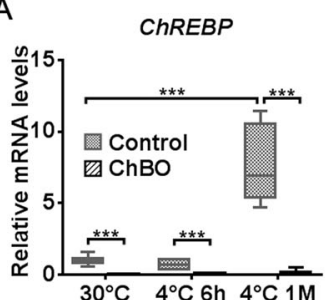

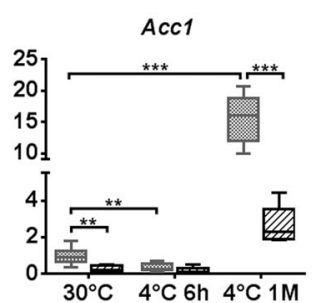

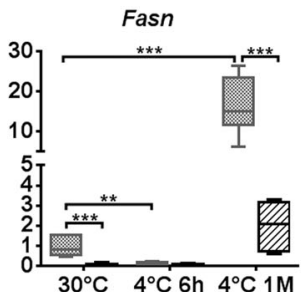

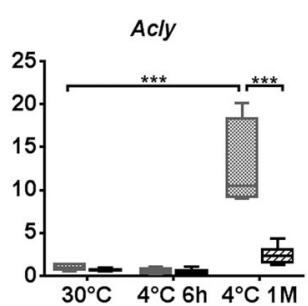

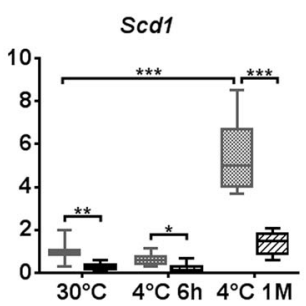

B

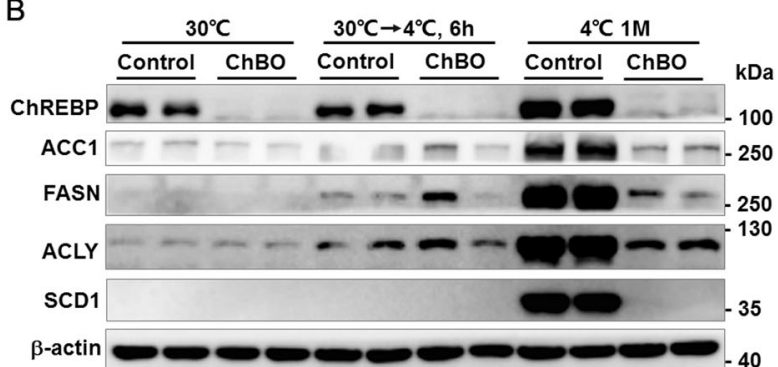

C

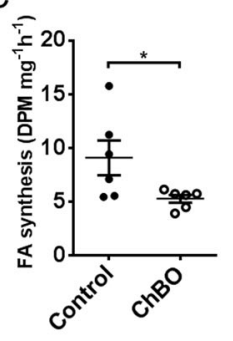

Fig. 6 Downregulated expression of DNL enzymes in BAT from ChBO mice. Chow-fed $\mathrm{ChBO}$ and control male mice housed at $30^{\circ} \mathrm{C}$ were subjected to $6 \mathrm{~h}$ of acute cold exposure or 1 month of chronic cold acclimation at $4{ }^{\circ} \mathrm{C}$. A mRNA levels of ChREBP, Acc1, Fasn, Scd1, and Acly genes in BAT ( $n=6-8$ per group). B Protein levels of DNL enzymes in BAT from ChBO and control mice $(n=4$ per group). C In vivo BAT lipogenesis was determined by measuring the incorporation of ${ }^{3} \mathrm{H}-\mathrm{H}_{2} \mathrm{O}$ into fatty acids of the BAT from ChBO and control mice housed at $4{ }^{\circ} \mathrm{C}$ for 1 week ( $n=6$ per group). Data are presented as mean \pm SEM. ${ }^{*} p<0.05 ;{ }^{* *} p<0.01 ;{ }^{* *} p<0.001$.

In conclusion, ChREBP-regulated de novo lipogenesis regulates glucose metabolism, however, it is dispensable for BAT thermogenesis.

\section{REFERENCES}

1. Bluher M. Obesity: global epidemiology and pathogenesis. Nat Rev Endocrinol. 2019;15:288-98.

2. Singh GM, Danaei G, Farzadfar F, Stevens GA, Woodward M, Wormser D, et al. The age-specific quantitative effects of metabolic risk factors on cardiovascular diseases and diabetes: a pooled analysis. PLoS One. 2013;8:e65174.

3. Czernichow S, Kengne AP, Stamatakis E, Hamer M, Batty GD. Body mass index, waist circumference and waist-hip ratio: which is the better discriminator of cardiovascular disease mortality risk?: evidence from an individual-participant meta-analysis of 82864 participants from nine cohort studies. Obes Rev. 2011;12:680-7.

4. Lauby-Secretan B, Scoccianti C, Loomis D, Grosse Y, Bianchini F, Straif K, et al. Body fatness and cancer-viewpoint of the IARC Working Group. N Engl J Med. 2016;375:794-8.

5. Hill JO, Wyatt HR, Peters JC. Energy balance and obesity. Circulation. 2012;126:126-32.

6. Bartelt A, Bruns OT, Reimer $\mathrm{R}$, Hohenberg $\mathrm{H}$, Ittrich $\mathrm{H}$, Peldschus $\mathrm{K}$, et al. Brown adipose tissue activity controls triglyceride clearance. Nat Med. 2011;17:200-5.

7. van Marken Lichtenbelt WD, Vanhommerig JW, Smulders NM, Drossaerts JM, Kemerink GJ, Bouvy ND, et al. Cold-activated brown adipose tissue in healthy men. N Engl J Med. 2009;360:1500-8.

8. Virtanen $K A$, Lidell ME, Orava J, Heglind $M$, Westergren $R$, Niemi $T$, et al. Functional brown adipose tissue in healthy adults. N Engl J Med. 2009;360:1518-25.

9. Hanssen MJ, Hoeks J, Brans B, van der Lans AA, Schaart G, van den Driessche JJ, et al. Short-term cold acclimation improves insulin sensitivity in patients with type 2 diabetes mellitus. Nat Med. 2015;21:863-5.

10. Yoneshiro T, Aita S, Matsushita M, Kayahara T, Kameya T, Kawai Y, et al. Recruited brown adipose tissue as an antiobesity agent in humans. J Clin Invest. 2013;123:3404-8.

11. Braun K, Oeckl J, Westermeier J, Li Y, Klingenspor M. Non-adrenergic control of lipolysis and thermogenesis in adipose tissues. J Exp Biol. 2018;221.

12. Klingenspor $M$, Ebbinghaus $C$, Hulshorst $G$, Stohr $S$, Spiegelhalter F, Haas $K$, et al. Multiple regulatory steps are involved in the control of lipoprotein lipase activity in brown adipose tissue. J Lipid Res. 1996;37:1685-95.

13. Okada K, LeClair KB, Zhang Y, Li Y, Ozdemir C, Krisko Tl, et al. Thioesterase superfamily member 1 suppresses cold thermogenesis by limiting the oxidation of lipid droplet-derived fatty acids in brown adipose tissue. Mol Metab. 2016;5:340-51.

14. Fedorenko A, Lishko PV, Kirichok Y. Mechanism of fatty-acid-dependent UCP1 uncoupling in brown fat mitochondria. Cell. 2012;151:400-13.

15. Mottillo EP, Balasubramanian P, Lee YH, Weng C, Kershaw EE, Granneman JG. Coupling of lipolysis and de novo lipogenesis in brown, beige, and white adipose tissues during chronic beta3-adrenergic receptor activation. J Lipid Res. 2014;55:2276-86.

16. Sanchez-Gurmaches J, Tang Y, Jespersen NZ, Wallace M, Martinez Calejman C, Gujja S, et al. Brown fat AKT2 is a cold-induced kinase that stimulates ChREBPmediated de novo lipogenesis to optimize fuel storage and thermogenesis. Cell Metab. 2018;27:195-209 e6.

17. lizuka K, Bruick RK, Liang G, Horton JD, Uyeda K. Deficiency of carbohydrate response element-binding protein (ChREBP) reduces lipogenesis as well as glycolysis. Proc Natl Acad Sci USA. 2004;101:7281-6.

18. Wei C, Ma X, Su K, Qi S, Zhu Y, Lin J, et al. ChREBP-beta regulates thermogenesis in brown adipose tissue. J Endocrinol. 2020;245:343-56.

19. Shi JH, Lu JY, Chen HY, Wei CC, Xu X, Li H, et al. Liver ChREBP protects against fructose-induced glycogenic hepatotoxicity by regulating L-type pyruvate kinase. Diabetes. 2020;69:591-602.

20. Li L, Li B, Li M, Niu C, Wang G, Li T, et al. Brown adipocytes can display a mammary basal myoepithelial cell phenotype in vivo. Mol Metab. 2017;6:1198-211.

21. Liu G, Zhou L, Zhang H, Chen R, Zhang $Y, L i ~ L$, et al. Regulation of hepatic lipogenesis by the zinc finger protein Zbtb20. Nat Commun. 2017;8:14824.

22. Bond LM, Ntambi JM. UCP1 deficiency increases adipose tissue monounsaturated fatty acid synthesis and trafficking to the liver. J Lipid Res. 2018;59:224-36.

23. Katz LS, Xu S, Ge K, Scott DK, Gershengorn MC. T3 and glucose coordinately stimulate ChREBP-mediated Ucp1 expression in brown adipocytes from male mice. Endocrinology. 2018;159:557-69.

24. Abdul-Wahed A, Guilmeau S, Postic C. Sweet sixteenth for ChREBP: established roles and future goals. Cell Metab. 2017;26:324-41.

25. Kursawe R, Caprio S, Giannini C, Narayan D, Lin A, D'Adamo E, et al. Decreased transcription of ChREBP-alpha/beta isoforms in abdominal subcutaneous adipose tissue of obese adolescents with prediabetes or early type 2 diabetes: associations with insulin resistance and hyperglycemia. Diabetes. 2013;62:837-44.

26. Eissing L, Scherer T, Todter K, Knippschild U, Greve JW, Buurman WA, et al. De novo lipogenesis in human fat and liver is linked to ChREBP-beta and metabolic health. Nat Commun. 2013;4:1528.

27. Vijayakumar A, Aryal P, Wen J, Syed I, Vazirani RP, Moraes-Vieira PM, et al. Absence of carbohydrate response element binding protein in adipocytes causes systemic insulin resistance and impairs glucose transport. Cell Rep. 2017;21:1021-35. 
28. Herman MA, Peroni OD, Villoria J, Schön MR, Abumrad NA, Blüher M, et al. A novel ChREBP isoform in adipose tissue regulates systemic glucose metabolism. Nature. 2012;484:333-8.

29. Nuotio-Antar AM, Poungvarin N, Li M, Schupp M, Mohammad M, Gerard S, et al. FABP4-Cre mediated expression of constitutively active ChREBP protects against obesity, fatty liver, and insulin resistance. Endocrinology. 2015;156:4020-32.

\section{AUTHOR CONTRIBUTIONS}

CW designed and carried out the study, interpreted and analyzed data, and drafted the article. PW and ZX analyzed the expressions of thermogenic genes. QD, ML, SQ and J-HS performed animal studies. X-HM performed the immunochemistry studies. WJZ and A-JR designed and conceived the experiments, reviewed the data, edited the article, and approved the version to be published.

\section{FUNDING}

This work was supported by grants from the National Key Research and Development Project of China (2019YFA0802500), the National Natural Science Foundation of
China $(91857203,31730042,32100929)$, and the Collaborative Innovation Program of the Shanghai Municipal Health Commission (2020CXJQ01, 21ZR1477900).

\section{COMPETING INTERESTS}

The authors declare no competing interests.

\section{ADDITIONAL INFORMATION}

Supplementary information The online version contains supplementary material available at https://doi.org/10.1038/s41366-022-01082-7.

Correspondence and requests for materials should be addressed to An-Jing Ren or Weiping J. Zhang.

Reprints and permission information is available at http://www.nature.com/ reprints

Publisher's note Springer Nature remains neutral with regard to jurisdictional claims in published maps and institutional affiliations. 